\title{
Análisis de la relación entre flexibilidad en operaciones y performance empresarial mediante técnicas bibliométricas ${ }^{1}$
}

\author{
Ana María Serrano-Bedia*, María Concepción López-Fernández*, Marta Pérez-Pérez* \\ * Departamento de Administración de Empresas. Universidad de Cantabria. \\ Correo-e: serranoa@unican.es, lopezm@unican.es, perezpm@unican.es
}

Recibido: 20-07-2012; 2a version: 07-11-2012; Aceptado: 26-11-2012.

Cómo citar este artículo/Citation: Serrano-Bedia, A. M.; López-Fernández, M. C.; Pérez-Pérez, M. (2013). Análisis de la relación entre flexibilidad en operaciones y performance empresarial mediante técnicas bibliométricas. Revista Española de Documentación Científica, 36(4):e022. doi: http://dx.doi.org/10.3989/redc.2013.4.1007

Resumen: El propósito de este trabajo es realizar un análisis bibliométrico en el campo del management de operaciones, en concreto el tema que relaciona flexibilidad operativa y la performance empresarial, en el periodo 1981-2011. En el análisis se aplican tanto indicadores de actividad (cuantificación de autores, revistas o Ley de Lotka) como de relación (citas por autor y revista, co-citación entre autores y análisis de co-palabras) utilizando como fuente de datos el Social Sciences Citation Index de Web of Science. Los resultados proporcionados por los indicadores de actividad han puesto de manifiesto que se trata de un campo de estudio relativamente reciente, en el que se verifica el cumplimiento de la ley de Lotka, y en el que los autores y revistas más productivos no necesariamente coinciden con los más citados. El análisis de co-citas ha identificado los autores que constituyen el marco teórico consolidado de referencia. Por último, el análisis de co-palabras ha permitido identificar las temáticas de investigación que conforman el ámbito de estudio y su clasificación en ampliamente desarrolladas, periféricas especializadas, periféricas poco desarrolladas y emergentes, a las que se debe prestar atención para contribuir al desarrollo y consolidación del campo.

Palabras clave: Indicadores bibliométricos; ley de Lotka; co-citación de autores; co-palabras; flexibilidad operacional; performance; Social Sciences Citation Index.

\section{Relationship between operational flexibility and performance through bibliometric techniques}

Abstract: The purpose of this paper is to present a bibliometric analysis in the field of operations management, specifically the issue that relates operational flexibility with business performance during the period 1981-2011. The analysis applies indicators concerning both activity (quantification of authors, journals or Lotka's Law) and relationships (citations by author, journal co-citation between authors and co-word analysis) using the Web of Science's Social Sciences Citation Index as a data source. The results provided by the activity indicators show that it is a relatively recent field of study, which verifies compliance with the Lotka's Law, and where the most productive authors and journals do not necessarily coincide with the most cited ones. The co-citation analysis has identified authors of reference in the field. Finally, the co-word analysis has allowed the identification of the research topics comprising the area of study and classified into widely developed issues, specialized peripheral issues, peripheral underdeveloped issues, and emerging subject matters. These are the most relevant, in that they identify important current issues that are still poorly developed.

Keywords: Bibliometric indicators; Lotka's law; co-citation of authors; co-words; operational flexibility; performance; Social Sciences Citation Index.

Copyright: () 2013 CSIC. Este es un artículo de acceso abierto distribuido bajo los términos de la licencia Creative Commons Attribution-Non Commercial (by-nc) Spain 3.0. 


\section{INTRODUCCIÓN}

Este trabajo presenta una aplicación del análisis bibliométrico al campo del Management de Operaciones, donde este tipo de análisis constituye una innovación metodológica frente a las formas más clásicas de revisión de la literatura. En este sentido, una búsqueda utilizando como base las publicaciones contenidas en Social Sciences Citation Index (SSCI) revela la existencia de únicamente tres artículos que hayan efectuado análisis bibliométricos en este campo: (Pilkington y Liston-Heyes ,1999), (Pilkington y Fitzgerald, 2006) y (Pilkington y Meredith, 2009). Estos trabajos basan su fundamentación en la identificación de temáticas a través del análisis de co-citas y por lo que se refiere a su contenido ponen de manifiesto que el campo de Management de Operaciones ha experimentado una evolución importante en los últimos años. La misma se concreta en un desplazamiento desde una visión en la que predominan temas de investigación más tácticos y fragmentados, hacia una preocupación por temas más integrados y estratégicos, destacando en este sentido una nueva tendencia de investigación hacia cuestiones como las cadenas de suministro o la flexibilidad (Pilkington y Meredith, 2009).

En relación con la segunda de estas temáticas identificadas como actuales y crecientes por Pilkington y Meredith (2009), su interés radica en el hecho de que, desde un punto de vista estratégico, la flexibilidad es una necesidad derivada de la combinación de un mercado globalizado, con una intensa competencia y unos rápidos cambios tecnológicos, circunstancias todas ellas que exigen a las empresas una elevada capacidad de respuesta a los retos derivados de esta situación. La capacidad de liderar tales procesos afecta a las posibilidades de supervivencia de las empresas, así como a sus rendimientos, y está íntimamente relacionada con la habilidad para responder mejor y en mejores condiciones de tiempo, coste, esfuerzo o rendimiento a las demandas del mercado con productos y/o servicios innovadores, de calidad y ofrecidos a un precio adecuado, dentro del marco de la estrategia competitiva de la empresa. Esta problemática general está estrechamente vinculada con el área de operaciones de la empresa, ámbito en cuyo desarrollo la flexibilidad desempeña un papel esencial en el logro de ventajas competitivas (Suarez y otros, 1996), (Beach y otros, 2000), (Slack, 2005). Por tanto el estudio de la relación entre flexibilidad operacional y performance empresarial resulta de interés desde una doble perspectiva, académica y profesional, y es por ello que el análisis de la literatura que ha investigado dicha relación, concretamente a partir de técnicas bibliométricas, constituye el objetivo principal de este trabajo.

El mismo presenta dos novedades principales frente a la literatura previa. En primer lugar, la derivada de la no existencia hasta la fecha de trabajos bibliométricos que hayan realizado un análisis pormenorizado de la relación entre la flexibilidad operacional y la performance. La segunda novedad radica en la incorporación al análisis no sólo de los indicadores más habituales de actividad y de relación de primera generación, sino también de segunda generación, y en concreto el análisis de co-palabras. Este último tipo de indicadores reviste especial interés para el investigador, por cuanto al centrarse en el análisis del contenido de los artículos para definir y clasificar temáticas de investigación permiten obtener una visión del campo científico disminuyendo el grado de componente subjetivo que acompaña a los procesos de revisión de la literatura tradicionales. A pesar de lo anterior, su presencia en el campo del Management de Operaciones es prácticamente inexistente -se ha identificado un único trabajo, (Sungjoo y otros, 2009), donde los autores proponen un enfoque para la creación y la utilización de mapas de palabras clave basadas en patentes para su uso en la nueva actividad de creación de tecnología-, por lo que el trabajo que aquí se presenta puede constituir una aportación relevante en este campo de estudio.

En base a estas consideraciones previas, el objetivo principal de este trabajo es realizar un estudio de la relación entre la flexibilidad operacional y la performance empresarial a través de técnicas bibliométricas. De una forma más precisa, los objetivos específicos del trabajo se concretan en los siguientes puntos:

1.- Aplicación de indicadores de actividad, a fin de obtener datos acerca del volumen e impacto de las actividades de investigación, así como sobre la distribución de los autores según su productividad. En particular, los indicadores seleccionados en el trabajo son el análisis de autores y revistas más productivas, evolución del campo de estudio y ley de Lotka.

2.- Aplicación de indicadores de relación de primera y segunda generación. Los primeros rastrean las conexiones entre investigadores y campos, mientras los segundos se centran en el análisis del contenido de los artículos para definir temáticas de forma clara. En particular, en este trabajo se emplea la cocitación de autores dentro del primer grupo, y el análisis de co-palabras dentro del segundo.

\section{MATERIAL Y MÉTODOS}

En este apartado se expondrá en primer lugar la metodología empleada en el proceso de revisión sistemática hasta obtener la muestra final de documentos para la realización del análisis bibliométrico. En segundo lugar, se expondrá brevemente el tipo de indicadores bibliométricos empleados, deteniéndonos en mayor medida en el análisis de los indicadores de segunda generación.

Para el desarrollo de la investigación se ha realizado una búsqueda sistemática previa accediendo 
a la base de datos ISI Web of Knowledge's Social Sciences Citation Index (SSCI) durante el mes de Julio de 2011 con los criterios que a continuación se detallan. El límite temporal seleccionado fue el máximo permitido (1981-2011) realizándose la búsqueda sistemática de referencias con las palabras clave cuya justificación se detalla a continuación:

1. Términos que definen el campo general de estudio (manufacturing flexibility y operational flexibility) al haber recaído la denominación de esta área funcional en cualquiera de los dos términos utilizados.

2. Términos que definen las dimensiones de flexibilidad de las áreas vinculadas en su totalidad al área de operaciones siguiendo la definición aportada por Aggarwal (1997). Así se incluyen las dimensiones product flexibility, volume flexibility, mix flexibility y machine flexibility.

3. Términos que definen la mano de obra como recurso del área de operaciones: incluyéndose el término labor flexibility como un recurso más de dicha área funcional.

4. Términos de resultado: analizándose tanto la dimensión cuantitativa de la performance como su dimensión cualitativa al incluirse los términos productivity, performance, quality e innovation.

La búsqueda de datos en SSCI se ha llevado a cabo con las condiciones que se señalan en la tabla I donde las palabras clave se utilizaron como criterio de selección para el topic o tema (título, palabras clave o resumen).

En relación a las condiciones de búsqueda cabe decir que se efectuó una comprobación del lenguaje de publicación de las revistas JCR clasificadas en las categorías Management y Operations Research que puso de manifiesto que el $96.16 \%$ de las revistas tenían como idioma de publicación el inglés, por lo que se eligió éste como lenguaje de búsque- da. En relación al tipo de documento, se decidió seleccionar los artículos y revisiones publicados en revistas como base para el análisis. Ambas fuentes no sólo constituyen la fuente de conocimiento más actual sino que además, autores como Holsapple y Lee- Post (2010) sostienen que las publicaciones en revistas son las que los investigadores del management de operaciones utilizan como conductos para el suministro de conocimientos a otros, siendo las revistas las más buscadas por los consumidores del management de operaciones.

Con estos criterios de búsqueda se obtuvo una muestra inicial de 237 documentos que tras un proceso de filtrado a partir de la lectura de los abstracts para eliminar errores se redujo a 128 documentos que podrían haber contribuido al desarrollo de la teoría o bien a probar la teoría en este campo. Obtenida la muestra final de documentos se creó en una segunda fase una base de datos ACCESS con la información obtenida y que fue ajustada para poder realizar el análisis sin que los resultados fueran distorsionados. En este sentido se realizaron los ajustes propios de los errores procedentes de la descarga de los datos, comprobándose por ejemplo que las referencias a un mismo autor se realizasen de la misma forma u homogeneizando las keywords de los textos en cuanto a plurales y singulares.

Concluido el proceso de revisión sistemática se detallan a continuación los indicadores bibliométricos empleados en esta investigación:

En primer lugar se incluyen indicadores de actividad, en particular análisis de autores y revistas más productivas, evolución del campo de estudio o ley de Lotka. Esta última es también conocida como cuadrática inversa de la producción científica y establece que el número $(Y)$ de aquellos autores que han publicado una cantidad determinada de trabajos $(\mathrm{X})$ en un área de investigación a lo largo de varios años de actividad es una relación fija con un número constante de autores que han publicado uno o muy pocos artículos (Lotka, 1926). Se for-

Tabla I. Condiciones de la búsqueda de revisión sistemática

\begin{tabular}{|c|c|}
\hline Palabras clave & $\begin{array}{l}\text { ("operati* flexib*" or "manufactur* flexib*" or "product* flexib*" or "volume flexib*" or } \\
\text { "machine flexib*" or "mix flexib*"or "labo* flexib*") AND ("performance" or "productiv*" or } \\
\text { "quality" or "innovati*") }\end{array}$ \\
\hline Tipo de documento & "article" y "review" (pero no "book review"). \\
\hline Área temática & $\begin{array}{c}\text { Management or Operations Research Management Science or Business or Economics or } \\
\text { Industrial Relations Labor or Business Finance sin ningún tipo de restricciones de selección } \\
\text { adicional. }\end{array}$ \\
\hline
\end{tabular}

FUENTE: Elaboración propia 
mula como $\mathrm{Y}=\mathrm{K} / \mathrm{X}^{\mathrm{n}}$ donde $\mathrm{K}$ y $\mathrm{n}$ son constantes $\mathrm{Y}$ generalmente $\mathrm{n}$ igual a 2 .

En segundo lugar se analizan los indicadores de relación de primera y segunda generación, en particular co-citación de autores y análisis de copalabras, respectivamente.

El análisis de la co-citación de autores enfatiza la idea de que las citas conjuntas que contienen los artículos científicos constituyen una referencia clara en un campo de investigación, permitiendo identificar los documentos considerados como fundadores y los que contribuyen a desarrollar el campo. De acuerdo con el trabajo de Sanz (2003), para medir la estructura, organización y nivel de integración de la red de co-citación se deben considerar dos aspectos: el primero consiste en la densidad del grafo, que muestra su alta o baja conectividad, siendo una medida expresada en porcentaje del cociente entre el número de relaciones existentes con el máximo de relaciones que podría tener si todos los nodos estuvieran conectados directamente con todos los demás (Sanz, 2003).

El segundo aspecto importante hace referencia a las medidas de centralidad, dentro de las cuales se pueden identificar según indica Freeman (1979) las de rango, cercanía y mediación. El rango mide el porcentaje de conexiones que tiene un nodo sobre el total de la red. La medida de centralidad basada en cercanía representa la independencia, la capacidad que tiene un nodo de alcanzar a los demás. Por último, la medida de mediación constituye el control de la comunicación de otros y su capacidad de restringirla, muestra cuando el actor es intermediario entre otros actores del mismo grupo que no se conocen entre sí. Para la representación del diagrama de co-citación de autores se utilizará el análisis de redes sociales (Social Network Analysis).

El análisis de co-palabras, por su parte, se basa en un principio simple: una especialidad de investigación puede ser identificada por su propio vocabulario, o más exactamente, por las particulares asociaciones que se establecen entre sus palabras clave (Callon y otros, 1993). Al contabilizar todas las apariciones conjuntas y representar sus relaciones gráficamente, es posible identificar distintas agrupaciones o clusters temáticos en los que la fuerza de la unión de las palabras que los integran se mide por un índice normalizado cuyo valor depende tanto de la aparición de las palabras individualmente como de sus apariciones conjuntas y que se calcula como:

$$
e_{i j}=\frac{c_{i j}^{2}}{c_{i} c_{j}}
$$

donde $\mathrm{C}_{\mathrm{ij}}$ mide la intensidad de asociación entre las dos palabras i y j, y $C_{\text { y }} \mathrm{C}_{\mathrm{j}}$ la frecuencia absoluta de aparición de las palabras i y j respectivamente.
Identificar las temáticas presentes en base al análisis de co-palabras constituye una primera etapa que debe ser completada con un análisis de las posiciones respectivas. Las nociones de centralidad y densidad permiten representar de forma sintética y simplificada la morfología de la red. Como cada cluster se define por su centralidad y densidad es posible trazar un diagrama estratégico donde:

Los agregados del cuadrante 1 (esquina superior derecha de la matriz) son a la vez centrales en la red general (están sólidamente conectados a otros agregados) y están recorridos por intensas relaciones que manifiestan su alto grado de desarrollo y de integración.

Los agregados del cuadrante 2 (esquina inferior derecha) son centrales, es decir, están ampliamente conectados a otros agregados, pero la densidad de sus relaciones internas es relativamente débil. Son susceptibles de convertirse en centrales y desarrollados, por lo que resultan importantes para el desarrollo del campo.

Los agregados del cuadrante 3 (esquina superior izquierda) son periféricos y la intensidad de sus relaciones internas (gran densidad) indica que se corresponden con problemáticas de investigación cuyo estudio está bien desarrollado. Puede tratarse de agregados que se han visto marginados progresivamente, atrayendo cada vez menos interés a su alrededor: aparecen como especializaciones que interactúan débilmente con respecto a las otras subredes.

Los agregados del cuadrante 4 (esquina inferior izquierda) son a la vez periféricos y poco desarrollados. Representan los márgenes de la red.

Por lo que se refiere a las herramientas utilizadas para el cálculo de estos indicadores, todos ellos son softwares de fácil acceso puestos a disposición de toda la comunidad científica. En relación a los indicadores de actividad y de relación de primera generación se utilizó el software SITKIS junto a los programas UCINET y NETDRAW, mientras que para el análisis de los indicadores de segunda generación (análisis de co-palabras) se ha utilizado el software específico REDES 2005, que ha permitido la identificación de las temáticas presentes en el campo y su clasificación de acuerdo a la matriz estratégica. El programa REDES requiere definir una serie de parámetros a través de los cuáles se obtienen tres resultados: la matriz de co-ocurrencia normalizada, la matriz de adyacencia y las agrupaciones por temáticas clasificadas en la matriz estratégica. En concreto, los parámetros definidos en este trabajo fueron: a) aparición mínima de palabras (cuatro); b) co-ocurrencia mínima de palabras (dos) y c) tamaño mínimo del grupo (tres).

La elección de los valores para estos parámetros se justifica siguiendo los pasos previos de Benavides y otros (2011). Por lo que se refiere al primero, aparición mínima de palabras, se ha optado por re- 
presentar las palabras clave que aparecen en más de tres artículos por dos razones. En primer lugar, para recoger aquellas palabras más relevantes en el campo (seleccionando aquellas de mayor repetición) y en segundo lugar para facilitar la lectura e interpretación de los resultados obtenidos. Continuando por el segundo, co-ocurrencia mínima de palabras, se especifica un valor de dos para garantizar la existencia de una línea de investigación, al encontrarse dos palabras ligadas en más de un texto científico. Por último, se especifica un tamaño mínimo del grupo de tres para que las agrupaciones o clusters resultantes tengan la suficiente consistencia temática.

\section{RESULTADOS}

En primer lugar se exponen los principales resultados obtenidos de la aplicación de los indicadores de actividad (evolución del campo de estudio, cumplimiento de la ley de Lotka, autores y revistas más productivas) para en segundo lugar comentar los resultados obtenidos de los indicadores de relación (co-citación de autores y análisis de co-palabras).

\section{Resultados indicadores de actividad}

Como ya se ha explicado anteriormente los resultados proporcionados por los indicadores de actividad proporcionan datos acerca del volumen y del impacto de las actividades de investigación, permitiendo observar la evolución cuantitativa de la literatura. Los resultados obtenidos en el estudio se presentan a continuación:

\section{a) Evolución del campo de estudio}

El análisis efectuado muestra un campo de estudio relativamente reciente, puesto que los primeros documentos datan de principios de los años 90. Su evolución ha constatado la existencia de distintos ciclos, aunque se observa con claridad una tendencia alcista de esta línea de investigación con ciertas caídas que identifican cuatro periodos fundamen- talmente suscitados por el cambio de tendencia temática entre cada uno de ellos (ver figura 1 ).

Así, el primer periodo identificado comprende de 1990 hasta 1997. En él mayoritariamente los trabajos se han centrado en dos temas centrales, por un lado en el papel que juega la flexibilidad como estrategia de manufactura y por otro, en el estudio de los Sistemas de Fabricación Flexibles (FMS o Flexible Manufacturing Systems) como respuesta ante el entorno competitivo. El objetivo principal era el desarrollo de modelos matemáticos y la medición objetiva de la flexibilidad. El segundo periodo o ciclo de investigación se extiende desde 1998 hasta 2001. En esta etapa predominan trabajos que analizan cuestiones centradas en las ventajas del rendimiento de la flexibilidad laboral junto a la flexibilidad de máquina, a la vez que existe un auge creciente de investigación por analizar el efecto que las tecnologías de la información pueden provocar en la relación flexibilidad-performance. El tercer periodo abarca desde 2002 hasta 2008. En este periodo parece continuarse con el estudio de líneas previas como son el encaje estratégico de la flexibilidad con el entorno (que parece adquirir su mayor auge en este periodo temporal) o el estudio de las tecnologías avanzadas de fabricación. Asimismo, también surge la preocupación por la investigación y el análisis de la cadena de suministro y su gestión así como un incipiente interés por la personalización en masa, cuya investigación aparece mayoritariamente en este intervalo de tiempo. Por último, el cuarto y último periodo se extiende desde 2009 hasta la actualidad. En él se vislumbra una mayor preocupación por la minimización de recursos y los problemas relacionados con la capacidad de inversión analizándose en mayor medida las configuraciones de recursos más eficientes para la empresa. Esta evolución se encuentra en plena sintonía con el contexto actual de crisis económica en que nos encontramos, y que está propiciando aún más el estudio sobre qué tipo de medidas se pueden llegar a tomar en una organización (más concretamente en el área de operaciones) para

Figura 1. Evolución de la investigación científica en el campo de operaciones

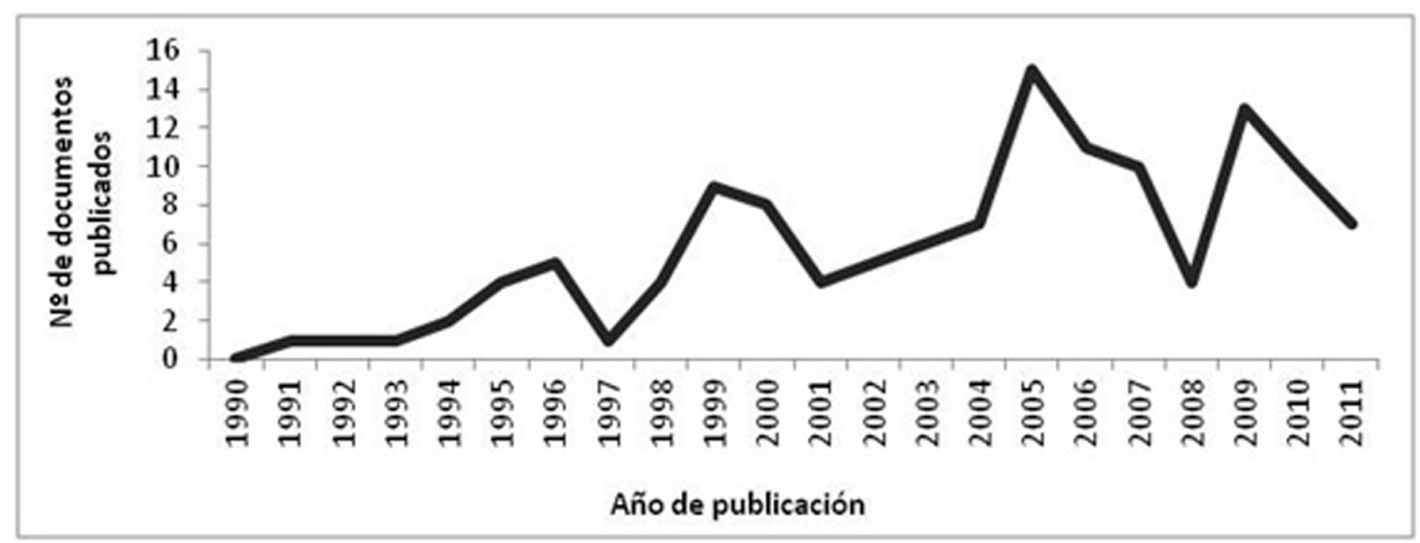


conseguir que las empresas aumenten su productividad y su competitividad.

Se debe matizar que los datos del último periodo hay que tomarlos con mucha cautela puesto que la búsqueda bibliográfica se realizó a principios de Julio de 2011, lo que implica que los datos completos de ese año probablemente no se obtendrán hasta mayo-junio de 2012.

\section{b) Cumplimiento de la Ley de Lotka}

Se ha efectuado un cómputo de artículos por autor para verificar en qué medida se cumple la ley de Lotka. En la literatura sobre la flexibilidad del área de operaciones durante el periodo 19912011 , $n$ toma un valor de 2.84, lo que evidencia que la ley de Lotka se verifica de forma bastante aproximada (ver figura 2). El que en esta área de estudio el valor sea superior a 2 pone de manifiesto que, en comparación con otras disciplinas (como por ejemplo el campo de la minería de datos cuyo valor es de 3.629 (Tsai, 2012), existe una mayor concentración de artículos en pocos autores productivos. El cumplimiento de esta ley es similar, en cambio, a las áreas de empresa familiar (Benavides y otros, 2011) o de la business ethic literature (Talukdar, 2011) donde los valores de $n$ alcanzan 2.68 y 2.6 respectivamente. Según los resultados, un total de 230 autores diferentes han escrito estos 128 artículos, de los cuales 185 sólo han publicado un texto ( $80.4 \%$ del total).

\section{c) Autores más productivos del campo de estudio}

Se ha analizado la productividad de los autores de la muestra y se ha comparado con el número de citas que han recibido cada uno de ellos dentro de la misma. En la muestra un total de 230 autores han escrito los 128 documentos (1,79 autores por artículo) y han recibido 1.999 citas. Mediante la tabla II se evidencia que no siempre los autores más productivos son los más citados. En ocasiones un solo trabajo puede ser tan relevante que aglutina el mayor número de citas (véase el caso de Gerwin). Atendiendo a esto, se ha calculado el promedio de citas recibidas por artículo y se ha procedido a presentar la información ordenada en base a esta variable en la tabla II.

Figura 2. Ley de Lotka en el campo de operaciones

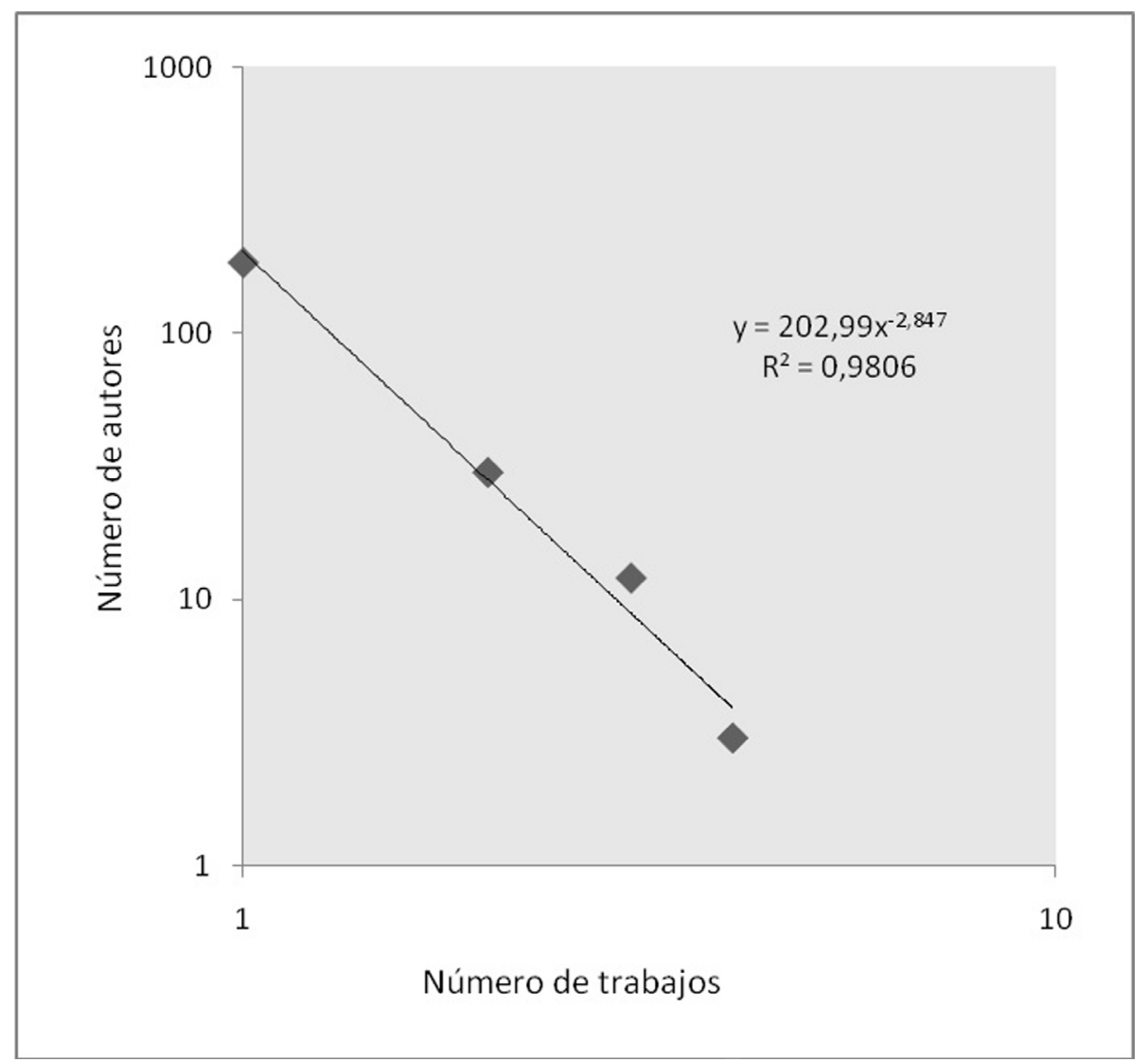


Tabla II. Comparativa entre productividad por autor y citas recibidas

\begin{tabular}{|c|c|c|c|c|c|c|c|}
\hline Autor & Productividad & Citas & $\begin{array}{l}\text { Promedio } \\
\text { por artículo }\end{array}$ & Autor & Productividad & Citas & $\begin{array}{l}\text { Promedio } \\
\text { por artículo }\end{array}$ \\
\hline Gerwin D & 1 & 36 & 36,00 & Das A & 2 & 3 & 1,50 \\
\hline Slack N & 2 & 33 & 16,50 & Suresh NC & 2 & 3 & 1,50 \\
\hline Benjaafar S & 1 & 12 & 12,00 & Molleman E & 3 & 4 & 1,33 \\
\hline Chen IJ & 1 & 9 & 9,00 & Holweg M & 2 & 2 & 1,00 \\
\hline Malhotra MK & 1 & 9 & 9,00 & Kathuria R & 2 & 2 & 1,00 \\
\hline Ward PT & 1 & 9 & 9,00 & Mohamed Z & 2 & 2 & 1,00 \\
\hline Barad M & 1 & 7 & 7,00 & Bish EK & 1 & 1 & 1,00 \\
\hline Jack EP & 2 & 8 & 4,00 & Calantone RJ & 1 & 1 & 1,00 \\
\hline Lau Rsm & 1 & 4 & 4,00 & Chung $\mathrm{CH}$ & 1 & 1 & 1,00 \\
\hline Lee $\mathrm{Hf}$ & 1 & 4 & 4,00 & Cousens A & 1 & 1 & 1,00 \\
\hline Vickery SK & 1 & 4 & 4,00 & De Treville S & 1 & 1 & 1,00 \\
\hline Stecke KE & 2 & 7 & 3,50 & Francas D & 1 & 1 & 1,00 \\
\hline De Toni A & 2 & 6 & 3,00 & Fry TD & 1 & 1 & 1,00 \\
\hline Anand G & 1 & 3 & 3,00 & Goyal M & 1 & 1 & 1,00 \\
\hline Bengtsson J & 1 & 3 & 3,00 & Hanna MD & 1 & 1 & 1,00 \\
\hline Felan JT & 1 & 3 & 3,00 & $\mathrm{Ho} \mathrm{CF}$ & 1 & 1 & 1,00 \\
\hline Jensen JB & 1 & 3 & 3,00 & Hong JD & 1 & 1 & 1,00 \\
\hline Koste LI & 1 & 3 & 3,00 & Iravani SM & 1 & 1 & 1,00 \\
\hline Oke A & 1 & 3 & 3,00 & Ketokivi M & 1 & 1 & 1,00 \\
\hline Sarker BR & 1 & 3 & 3,00 & Raman N & 1 & 1 & 1,00 \\
\hline Browne S & 1 & 2 & 2,00 & Roth AV & 1 & 1 & 1,00 \\
\hline Camison C & 1 & 2 & 2,00 & Safizadeh MH & 1 & 1 & 1,00 \\
\hline Cusumano MA & 1 & 2 & 2,00 & Salvador F & 1 & 1 & 1,00 \\
\hline Das SR & 1 & 2 & 2,00 & Chang SC & 3 & 2 & 0,67 \\
\hline Karuppan CM & 1 & 2 & 2,00 & Olhager $\mathrm{J}$ & 3 & 1 & 0,33 \\
\hline Krajewski LJ & 1 & 2 & 2,00 & Swink M & 4 & 1 & 0,25 \\
\hline Nembhard DA & 1 & 2 & 2,00 & Llorens FJ & 4 & 1 & 0,25 \\
\hline Suarez FF & 1 & 2 & 2,00 & Verdu AJ & 4 & 1 & 0,25 \\
\hline Wadhwa S & 1 & 2 & 2,00 & Garcia-Morales VJ & 4 & 1 & 0,25 \\
\hline Narasimhan R & 3 & 5 & 1,67 & & & & \\
\hline
\end{tabular}

FUENTE: Elaboración propia 
Además debe destacarse que existen otros autores que han sido citados en gran número de ocasiones por el resto de artículos de la muestra, lo que sugiere que constituyen autores de referencia en el campo de operaciones y relevantes a la hora de iniciar una investigación en este campo.

\section{d) Revistas más productivas}

Los 128 documentos que integran la muestra final han sido publicados por 30 revistas diferentes. En total estos artículos han citado a 757 revistas. En la tabla III se presenta el número de documentos que ha publicado cada revista dentro de la muestra para analizar cuál es la más productiva, comparándose con el número de citas que recibe la revista. Asimismo, tal y como se realizó en el caso de los autores se ha calculado el promedio de citas recibidas por artículo y se ha ordenado la información en torno a esta variable. Así, por ejemplo, llama la atención que las revistas más productivas son la International Journal of Operations \& Production Management y la International Journal of Production Research aglutinando entre las dos el $31,25 \%$ de la producción de artículos. Sin embargo estas dos revistas no son las más destacadas en cuanto a promedio de citas donde destacan la Management Science junto con la Journal of Operations Management, lo que evidencia la importancia de los artículos publicados en las mismas.

Tabla III. Comparativa entre revistas más productivas y citas recibidas por revista

\begin{tabular}{|c|c|c|c|}
\hline Revista & $\begin{array}{l}\mathrm{N}^{\circ} \text { de } \\
\text { documentos } \\
\text { publicados }\end{array}$ & $\begin{array}{l}\text { No de citas } \\
\text { recibidas } \\
\text { dentro de la } \\
\text { muestra }\end{array}$ & $\begin{array}{l}\text { Promedio citas } \\
\text { por artículo }\end{array}$ \\
\hline Management Science & 5 & 120 & 24,00 \\
\hline Journal of Operations Management & 6 & 88 & 14,67 \\
\hline Journal of Product Innovation Management & 1 & 7 & 7,00 \\
\hline Operations Research & 1 & 7 & 7,00 \\
\hline Industrial Marketing Management & 1 & 5 & 5,00 \\
\hline European Journal of Operational Research & 8 & 39 & 4,88 \\
\hline Decision Science & 7 & 34 & 4,86 \\
\hline IEEE Transactions on Engineering Management & 3 & 13 & 4,33 \\
\hline International Journal of Operations \& Production Management & 21 & 82 & 3,90 \\
\hline International Journal of Production Research & 19 & 67 & 3,53 \\
\hline International Journal of Production Economics & 8 & 27 & 3,38 \\
\hline IIE Transactions & 3 & 10 & 3,33 \\
\hline Omega & 9 & 27 & 3,00 \\
\hline African Journal of Business Management & 1 & 3 & 3,00 \\
\hline Journal of Small Business Management & 1 & 3 & 3,00 \\
\hline International Journal of Technology Management & 2 & 5 & 2,50 \\
\hline Journal of Management Studies & 2 & 5 & 2,50 \\
\hline International Journal of Flexible Manufacturing Systems & 3 & 6 & 2,00 \\
\hline Production and Operations Management & 7 & 12 & 1,71 \\
\hline Production Planning \& Control & 6 & 6 & 1,00 \\
\hline Work and Occupations & 1 & 1 & 1,00 \\
\hline Journal of the Operational Research Society & 1 & 1 & 1,00 \\
\hline International Journal of Computer Integrated Manufacturing & 1 & 1 & 1,00 \\
\hline Technovation & 1 & 1 & 1,00 \\
\hline British Journal Management & 1 & 1 & 1,00 \\
\hline Journal of Management Information Systems & 1 & 1 & 1,00 \\
\hline Journal of Engineering and Technology Management & 2 & 1 & 0,50 \\
\hline Manufacturing and Service Operations Management & 4 & 1 & 0,25 \\
\hline Revista Española de Financiación y Contabilidad & 1 & - & 0,00 \\
\hline Total Quality Management and Business Excellence & 1 & - & 0,00 \\
\hline
\end{tabular}

FUENTE: Elaboración propia 


\section{Resultados indicadores de relación}

\section{a) Red de co-citación entre autores}

Tal y como se ha expuesto en el apartado de material y métodos, de acuerdo con el trabajo de Sanz (2003) para medir la estructura y organización de la red y el nivel de integración se deben considerar dos aspectos: la densidad del grafo y las medidas de centralidad. En nuestro caso, el grafo está formado por 332 relaciones de entre las 380 relaciones máximas. En este sentido, su densidad es de $87.36 \%$, es decir, los actores están bastante relacionados.

Asimismo en cuanto al segundo de los aspectos del grafo se presenta el asociado a la medida de rango (figura 3). Asimismo, y para facilitar la lectura se incluye una tabla comparativa con los valores de los nodos según las tres medidas de centralidad posibles (tabla IV) a fin de poder efectuar comparaciones.
Analizando la centralidad a través del rango, se puede apreciar la existencia de varios artículos que permiten acceder a la mayor información que se establece entre la red de citas, pero sin determinar la importancia de esta información. Es decir el grado nodal define la influencia directa de un nodo, determinada por lo bien conectado que esté. En este caso concreto destaca el artículo de Gerwin (1987) el cual posee el mayor número de lazos directos con otros artículos. A este documento le siguen otros como Slack (1983), y Browne y otros (1984) que también constituyen artículos de revisión de la flexibilidad de manufactura. El artículo con menor centralidad de rango lo constituye Kogut y Kulatilaka (1994) y representa el menor número de contactos directos con otros artículos. Los valores de los nodos principales se muestran en la tabla IV junto a los valores de las otras dos medidas de centralidad cuyos grafos no se representan para facilitar la lectura de la información.

Figura 3. Red de co-citación de autores según el rango (degree)

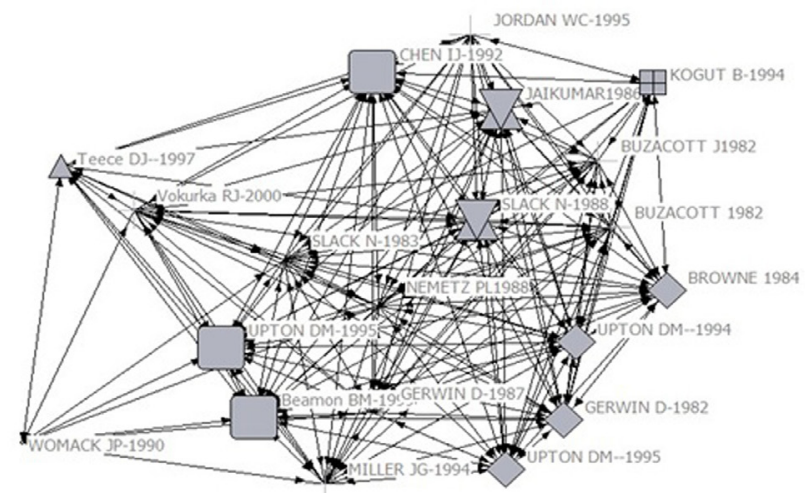

Tabla IV. Artículos de referencia ordenados por importancia en rango, cercanía y mediación

\begin{tabular}{cccccc}
\hline \multicolumn{2}{c}{ RANGO - Media 12,142 } & \multicolumn{2}{c}{ CERCANÍA - Media 78,856 } & \multicolumn{2}{c}{ MEDIACIÓN - Media 1,2025 } \\
\hline Autor & Valor & Autor & Valor & Autor & Valor \\
\hline Gerwin D 1987 & 30.154 & Gerwin D 1987 & 100.000 & Gerwin D 1987 & 3.434 \\
\hline Slack N 1983 & 27.692 & Slack N 1983 & 100.000 & Slack N 1983 & 3.434 \\
\hline Browne J 1984 & 25.538 & Jaikumar R 1986 & 92.593 & Jaikumar R 1986 & 2.679 \\
\hline Jaikumar R 1986 & 22.154 & Stigler G 1939 & 89.286 & Chen IJ 1992 & 2.076 \\
\hline Upton DM 1994 & 17.231 & Chen IJ 1992 & 89.286 & Stigler G 1939 & 1.888 \\
\hline Chen IJ 1992 & 17.231 & Upton DM 1994 & 86.207 & Browne J 1984 & 1.866 \\
\hline Gerwin D 1982 & 15.692 & Browne J 1984 & 86.207 & Nemetz PL 1988 & 1.685 \\
\hline Buzacott JA 1982 & 13.538 & Nemetz PL 1988 & 86.207 & Upton DM 1994 & 1.543 \\
\hline Stigler G 1939 & 11.692 & Buzacott JA1982 & 80.654 & Buzacott JA 1982 & 1.290 \\
\hline Slack N 1988 & 11.385 & Jordan WC 1995 & 80.645 & Gerwin D 1982 & 1.178 \\
\hline Nemetz PL 1988 & 10.462 & Gerwin D 1982 & 80.645 & Jordan WC 1995 & 0.899 \\
\hline Jordan WC 1995 & 10.154 & Slack N 1988 & 75.758 & Upton DM 1995 & 0.867 \\
\hline Upton DM 1995 & 9.231 & Upton DM 1995 & 75.758 & Slack N 1988 & 0.820 \\
\hline Teece DJ 1997 & 8.615 & Upton DM July 1995 & 73.529 & Miller JG 1994 & 0.772 \\
\hline Upton DM 1995 & 8.308 & Miller JG 1994 & 71.429 & Beamon BM 1999 & 0.579 \\
\hline Vokurka RJ 2000 & 8.000 & Teece DJ 1997 & 69.444 & Vokurka RJ 2000 & 0.455 \\
\hline Buzacott JA1982 & 6.154 & Kogut B 1994 & 69.444 & Womack JP 1990 & 0.404 \\
\hline Miller JG 1994 & 5.538 & Vokurka RJ 2000 & 69.444 & Teece DJ 1997 & 0.374 \\
\hline Beamon BM 1999 & 5.538 & Beamon BM 1999 & 67.568 & Upton DM July 1995 & 0.307 \\
\hline Womack JP 1990 & 5.538 & Womack JP 1990 & 67.568 & Kogut B 1994 & 0.224 \\
\hline Kogut B 1994 & 4.615 & Buzacott JA 1982 & 60.976 & Buzacott JA 1982 & 0.024 \\
\hline F 1924
\end{tabular}

FUENTE: Elaboración propia 
Comparando los valores que proporciona la tabla IV podemos afirmar que el actor más cercano de la red de artículos más influyentes es el artículo de Gerwin (1987) seguido de Slack (1983). Son por tanto, los que representan un mayor nivel de actividad comunicativa dentro de la red, los que tienen más capacidad de acceder al resto de los artículos referenciados o los que más intermedian entre pares de documentos no relacionados entre sí.

\section{b) Análisis de co-palabras}

Este análisis ha permitido identificar y clasificar el conjunto total de artículos en temáticas claras, identificándose en base a la palabra que constituye el nodo central 6 clusters (Simulation, Capacity, Strategy, Resource Based View, Machine Flexibility y Management). Estas 6 temáticas han sido caracterizadas de acuerdo a los criterios de densidad y centralidad explicados al inicio construyéndose una matriz estratégica (figura 4) que los agrupa en distintos cuadrantes:

Un primer cuadrante -esquina superior derechadefine las temáticas ampliamente desarrolladas y centrales. Dentro del mismo se incluye el cluster Management (42.19\%) que aglutina mayoritariamente los trabajos de corte más teórico o exploratorio con dos orientaciones básicas. La primera, centrada en el desarrollo de modelos explicativos de la relación flexibilidad-performance, indicando posibles variables moderadoras en la misma -configuración de los recursos de manufactura, incertidumbre del entorno, incertidumbre competitiva o tecnológica entre otras-. La segunda, centrada en la identificación o discusión de las diversas di- mensiones que integran el constructo flexibilidad de manufactura, aunque sin llegar a un consenso sobre las mismas.

Un segundo cuadrante -esquina inferior derechadefine las temáticas emergentes susceptibles de ser investigadas para contribuir al desarrollo y consolidación del campo, con dos clusters dentro del mismo. El primero, el cluster Strategy (22.67\%), incluye trabajos que analizan los efectos que diversas variables internas y externas a la organización -estrategia o grado de customización, en el primer caso, y factores ambientales y gubernamentales, en el segundo- provocan en dos dimensiones de flexibilidad, concretamente la flexibilidad en volumen y la flexibilidad en producto o mix. En general, las relaciones entre ambos conjuntos de variables se han analizado de forma individual, sin incorporar los posibles efectos sinérgicos que pueden producirse entre ellas, y por lo que se refiere al sentido de dichas relaciones los resultados encontrados son contrapuestos aunque mayoritariamente positivos. El segundo es el cluster Resource Based View $(5.47 \%)$. En este caso, las investigaciones se han centrado en analizar fundamentalmente tres aspectos: la identificación de las variables moderadoras de la relación flexibilidad laboral e innovación -concretamente el entorno y la cooperación interorganizacional- ; el efecto que las capacidades dinámicas -capacidad de aprendizaje y know-howprovocan sobre la flexibilidad operacional, con un resultado mayoritariamente positivo, y por último el estudio de la tecnología como recurso soporte de la flexibilidad, limitándose en este último caso el análisis a la dimensión flexibilidad de producto.

Figura 4. Matriz estratégica: temáticas presentes en el área de operaciones

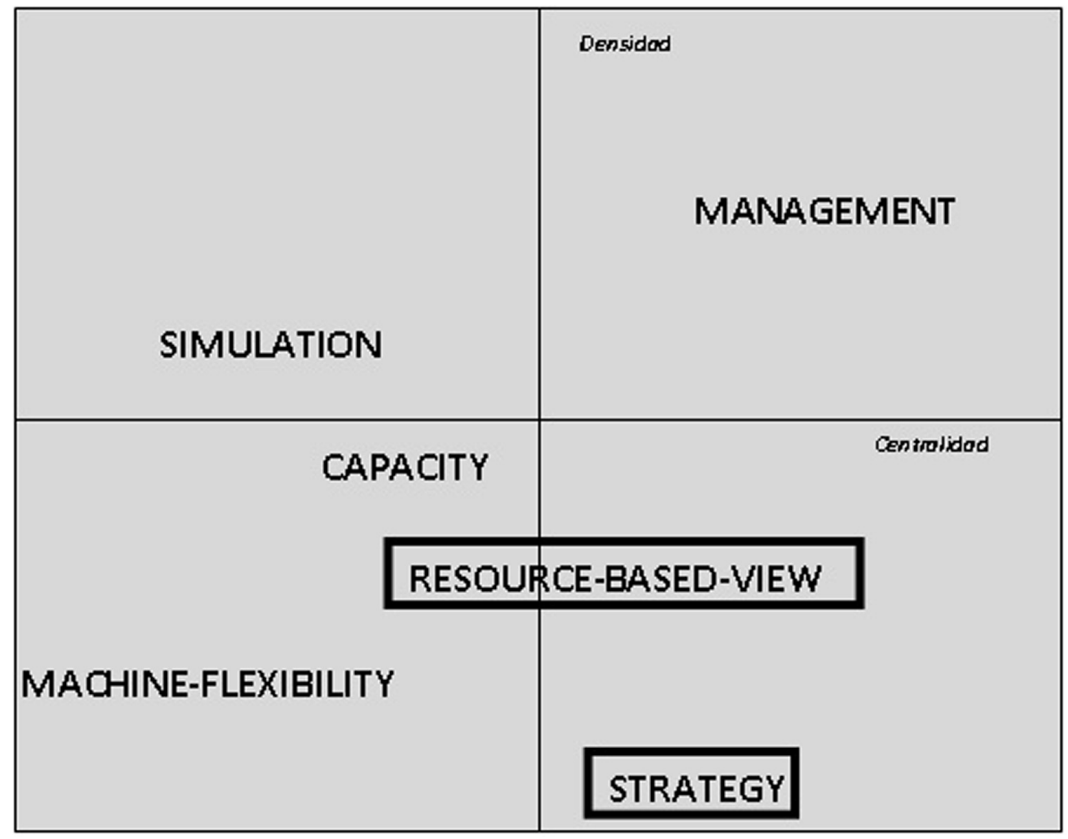


Un tercer cuadrante -esquina superior izquierda- define las temáticas periféricas y especializadas como el cluster Simulation $(11.7 \%$ de la muestra), el cual se centra en el análisis de la dimensión de flexibilidad asociada a los recursos humanos, y más concretamente a la determinación de las características de personal más adecuadas para satisfacer las necesidades de producción. En este caso se analiza el factor humano como recurso fundamental para la mejora de la competitividad de la organización desde una doble perspectiva: individual y organizativa, analizando la relación entre la flexibilidad en recursos humanos y otras dimensiones vinculadas a los recursos productivos, como los equipos, así como el efecto de prácticas de gestión de recursos humanos para mejorar la flexibilidad -como el entrenamiento cruzado, o la flexibilidad de la jornada de trabajosobre la performance.

Por último, un cuarto cuadrante -esquina inferior izquierda- define las temáticas periféricas y poco desarrolladas, encontrándose aquí en primer lugar el cluster Machine flexibility $(7.03 \%)$ que desarrolla mayoritariamente modelos matemáticos de medición del impacto sobre resultados que provocan los distintos sistemas de fabricación en general, y de manera particular los Sistemas de Fabricación Flexible (Flexible Manufacturing Systems, FMS). De una forma más precisa, los trabajos incluidos dentro de este cluster se han centrado en la relación existente entre la flexibilidad de máquinas individuales, o FMS en general, y la flexibilidad en ruta, confirmando una relación entre ambas. Asimismo han identificado algunos factores -como el número de máquinas en el proceso productivo o el proceso de planificación y control de la producción desarrollado- que pueden afectar de forma significativa a la relación entre flexibilidad de máquina y performance. En segundo lugar, este cuadrante incluye el cluster Capacity (10.94\%). En el mismo los trabajos utilizan de nuevo mayoritariamente modelos matemáticos y la investigación se encuentra fundamentalmente centrada en dos dimensiones de flexibilidad, concretamente la flexibilidad en volumen y su relación con la flexibilidad en producto o mix, encontrando resultados contrapuestos sobre su efecto combinado en la performance. También se presta atención a diferentes factores, tanto contextuales -incertidumbre del entorno, características y variabilidad de la demanda, ciclo de vida de los productos- como internos -proceso de asignación de pedidos, disponibilidad de recursos, grado de relación entre productos, estructura de costes, grado de customización del producto- que pueden afectar a la relación investigada.

\section{CONCLUSIONES}

Este trabajo ha presentado una aplicación concreta de las técnicas bibliométricas al campo que estudia la relación entre las dimensiones de flexibilidad del área de operaciones y la performance, utilizando como base las publicaciones contenidas en el WOS en un periodo de 30 años. En concreto del análisis efectuado se han derivado tres tipos de resultados principales.

Por lo que se refiere al primer objetivo específico, señalar que la información proporcionada por los indicadores de actividad ha puesto de manifiesto que se trata de un campo de estudio que está experimentando una evolución ascendente, y donde las temáticas emergentes tienen un componente más estratégico que operativo. Esta evolución es consistente con la experimentada por el campo de Management de Operaciones en general, tal y como ponen de manifiesto Pilkington y Meredith (2009). Asimismo se considera un campo de estudio relativamente reciente, puesto que los primeros documentos datan de principios de los años 90. En el mismo se verifica el cumplimiento de la ley de Lotka, lo que supone que hay una mayor concentración de artículos en pocos autores productivos en comparación con otras disciplinas, y en el que los autores y revistas más productivos no necesariamente coinciden con los más citados. Así, por ejemplo, las revistas más productivas son la International Journal of Operations \& Production Management y la International Journal of Production Research, mientras que las más destacadas en cuanto a promedio de citas son Management Science junto con Journal of Operations Management.

Continuando con el segundo de los objetivos específicos señalados, los resultados aportados por los indicadores de primera generación han permitido identificar los autores que constituyen el marco teórico de referencia, destacando en este sentido los artículos de Gerwin (1987), Slack (1983), Browne (1984) o Jaikumar (1986). Por último, los resultados de los indicadores de segunda generación han permitido identificar, y clasificar en una matriz estratégica, las temáticas que integran este campo. Por lo que a la identificación de temáticas se refiere, se han obtenido un total de 6 clusters: Simulation, Capacity, Strategy, Resource Based View, Machine Flexibility y Management, y en cuanto a su clasificación en la matriz estratégica se identifican temáticas ampliamente desarrolladas (Management), periféricas especializadas (Simulation), periféricas poco desarrolladas (Machine Flexibility y Capacity) y las emergentes (Resource Based View y Strategy). Por tanto, y desde una perspectiva global, puede afirmarse que el análisis efectuado ha permitido dar cumplimiento a los objetivos específicos planteados en el trabajo, llegando a describir un campo cuya estructura es compleja y rica, ya que encontramos todas las familias de los temas: unos centrales, otros periféricos, y diferentes grados de desarrollo, lo que sugiere una dinámica importante en el mismo.

Por lo que se refiere al objetivo principal del trabajo, esto es el estudio de la relación entre flexibilidad operacional y performance, el análisis del contenido de los diferentes clusters identificados mediante 
los indicadores de segunda generación revela que el grueso de la investigación desarrollada hasta la fecha ha abordado este problema desde una perspectiva parcial y una visión eminentemente táctica. Estas afirmaciones se justifican, en primer lugar, por cuanto la mayoría de los trabajos centran su interés tan sólo en una o, a lo sumo, dos de las dimensiones del constructo flexibilidad operacional. En segundo lugar, porque el análisis de tales dimensiones se realiza para casos o situaciones concretas, incorporando tan sólo en algunos trabajos un reducido número de variables internas y externas que pueden afectar a los resultados obtenidos.

En concordancia con lo anterior, el análisis realizado ha puesto de manifiesto que las futuras líneas de investigación de la relación entre flexibilidad operacional y performance deberían abordar el estudio de esta relación desde una perspectiva estratégica y empleando marcos teóricos plenamente aceptados y desarrollados en otros ámbitos del management, pero aún escasamente aplicados en este campo concreto, como la visión basada en los recursos y el enfoque de capacidades dinámicas.

\section{NOTAS}

[1] Una versión anterior a este trabajo fue presentada en el III Workshop on Operations Management and Technology celebrado en Cartagena en mayo de 2012. La presentación que sirvió de base para el mismo puede consultarse en http:// www.upct.es/ adem/omtech/programa/programa.html

\section{BIBLIOGRAFÍA}

Aggarwal, S. (1997). Flexibility management: the ultimate strategy. Industrial Management, vol. 39 (1), 26-31.

Beach, R.; Muhlemann, A.P.; Price, D.H.R.; Paterson, A.; Sharp, J.A. (2000). A review of manufacturing flexibility. European Journal of Operational Research, vol. 122 (1), 41-57.

Benavides Velasco, C.A.; Guzman Parra, V.F.; Quintana Garcia, C. (2011). The evolution of Family Firm literature as a research discipline. Cuadernos de Economía y Dirección de la Empresa, vol. 14 (2), 78-90.

Browne, J.; Dubois, D.; Rathmill, K.; Sethi, S.P.; Stecke, K.E. (1984). Classification of flexible manufacturing systems. The FMS magazine, vol. 2 (2), 114-117.

Callon, M.; Courtial, J.P.; Penan, H. (1993). Cienciometría. La medición de la actividad científica: de la bibliometría a la vigilancia tecnológica. Gijón: Ediciones Trea.

Freeman, L. C. (1979). Centrality in social networks conceptual clarification. Social networks, vol. 1 (3), 215-239.
Gerwin, D. (1987). An agenda for research on the flexibility of manufacturing processes. International Journal of Operations \& Production Management, vol. 7 (1), 38-49.

Holsapple,C.; Lee-Post, A. (2010). Behavior-based Analysis of Knowledge Dissemination Channels in Operations Management. The International Journal of Management Science, vol. 30 (1), 167-178.

Jaikumar, R. (1986). Postindustrial Manufacturing. Harward Business Review, vol. 64 (6), 69-76.

Kogut, B.; Kulatilaka, N. (1994). Operating flexibility, global manufacturing, and the option value of a multinational network. Management Science, vol. 40 (1), 123-139.

Lotka, A. J. (1926). The frequency distribution of scientific productivity. Journal of Washington Academy Sciences, vol. 16 (12), 317-323.

Pilkington, A.; Fitzgerald, R. (2006). Operations management themes, concepts and relationships: a forward retrospective of the IJOPM. International Journal of Operations and Production Management, vol. 26 (11), 1255-1275.

Pilkington, A.; Liston-Heyes, C. (1999). Is production and operations management a discipline? A citation/co-citation study. International Journal of Operations \& Production Management, vol. 19 (1), 7-20.

Pilkington, A. ; Meredith, J. (2009). The evolution of the intellectual structure of operations management 1980-2006: A citation/co-citation analysis. Journal of Operations Management, 27 (3), 185202.

Slack, N. (1983). Flexibility as a manufacturing objective. International Journal of Operations \& Production Management, vol. 3 (3), 4-13.

Sanz Menéndez, L. (2003). Análisis de Redes Sociales: o como representar las estructuras sociales subyacentes. Apuntes de Ciencia y Tecnología, vol. 7, 21-29.

Slack, N. (2005). The changing nature of operations flexibility. International Journal of Operations \& Production Management, vol. 25 (12), 1201-1210.

Suarez, F.F.; Cusumano, M.A.; Fine, C.H. (1996). Empirical study of manufacturing flexibility in printed circuit board assembly. Operations research, vol. 44 (1), 223-240.

Sungjoo, L.; Byungun, Y.; Yongtae, P. (2009). An approach to discovering new technology opportunities: Keyword-based patent map approach. Technovation, vol. 29 (6-7), 481-497.

Talukdar, D. (2011). Patterns of Research Productivity in the Business Ethics Literature: Insights from Analyses of Bibliometric Distributions. Journal of business ethics, vol. 98 (1), 137-151.

Tsai, H.H. (2012). Global data mining: An empirical study of current trends, future forecasts and technology diffusions. Expert systems with applications, vol. 39 (9), 8172-8181. 